\title{
DETERMINATION AND VISUALIZATION OF UNCERTAINTIES IN 4D-TRAJECTORY PREDICTION
}

\author{
Paul Weitz, German Aerospace Center (DLR), Braunschweig, Germany
}

\begin{abstract}
Future air traffic management (ATM) relies on trajectory forecasting for higher safety, efficiency, and environmental friendliness. The quality of trajectory predictions is subject to inaccuracies from a variety of sources [1]. This impreciseness in performance and environmental parameters cause inaccuracies in the calculations of flight trajectories. Therefore, the implementation of future trajectorybased air traffic management procedures requires decision support tools as well as reporting tools to analyze and predict uncertainties in actual flight paths. This paper presents a concept to detect uncertainties based on air traffic simulations, and to visualize the results in a quantitative (diagrams) as well as a qualitative way (geospatial imaging). For the representation of scalar errors, charts generated by JFreeChart-Tool [2] are suitable. NASA World Wind [3] is used for the geographical visualization of trajectories and their errors.

Within this paper we describe the process of evaluating the accuracy of a trajectory concerning the predicted flight path. Furthermore, the paper provides an overview of the most important factors for trajectory prediction, which are taken into account for the theoretical concept of the paper. The selected factors are meteorological parameters (wind, temperature), aircraft performance (weight, speed), and navigation performance. To compute the uncertainty of trajectory prediction we develop a metric that classifies the errors by four dimensions. For the investigation of the effect of parametric errors a simulation with over 2,000 flights under various environmental conditions has been implemented. For the validation of the method the study applies error analysis to the simulation results. With the developed tool it is possible to analyze the ramifications of uncertain prediction of trajectory on a single flight, as well as on the complex ATM system.
\end{abstract}

\section{Introduction}

Various aspects like separation or controller work load are reasons for capacity constraints [4]. The implementation of 4D-trajectory based ATM contains great potential for higher efficiency in capacity usage and lower delays. Additionally, an earlier detection of conflicts is feasible in trajectory forecasting [5]. To confirm the benefits, DLR's Institute of Flight Guidance conducts research with focus on trajectories based on simulation systems. These simulations are strongly focused on generating scientific data which are evaluated subsequent to the campaign. To be able to evaluate the simulations, we developed a method to calculate and visualize the uncertainty in trajectory prediction. The concept is implemented as a tool into DLR's evaluation environment "Extensible Workflow Management for Simulations" (EWMS) [6] and validated using simulation scenarios. The software system described in this paper is an extension to the reporting suite EWMS. The EWMSSoftware was developed to streamline and simplify simulation data management, significantly to reduce the effort required to analyze simulation results, and provides a large number of validated analysis algorithms [6].

The Following approaches evaluate uncertainty with respect to trajectory prediction: Mueller [7] developed a trajectory prediction uncertainty model. This error model is based on a linear error covariance analysis combined with linear control feedback. Based on this model particular flight phases can be analyzed but in the context of the ATM process evaluation of the whole flight is necessary. Wanke [4] considers empirical observations of uncertainty in sector demand predictions under current operational conditions, and on applying those measurements towards improving the performance and human factors of traffic flow management decision support systems. 
The display developed by Wanke visualizes the entry and exit point of a sector. Wanke's approach is focused on the trajectory in detail and the relation between two or more trajectories.

Hence our approach implements a tool to visualize the exact route within the bounds of uncertainty. Furthermore, the uncertainty is generated for future scenarios by traffic simulation. The approach for the visualization of uncertainties in trajectory prediction includes three tasks: generating simulation data, calculating uncertainties, and preparing results for diagrams or geospatial imaging. The uncertainty of an aircraft position can be described in terms of temporal or spatial metrics by three-dimensional ellipsoids. For the processing in computational systems the error data are classified and the possibilities of visualization in diagrams and geo systems are evaluated.

First trials confirm the assumption that the better the quality of forecast, the lesser the deviation of the actual flight from the prediction. This theory needs to be analyzed in this paper.

\section{Reporting Process}

The workflow of analyzing a conjecture is standardized in the context of air traffic simulations. The Reporting process stands for the process starting with the generation of a set of trajectories, followed by the evaluation of uncertainty and ends with the visualization of uncertainties. The paper presents a supporting tool for the visualization of uncertainties and the effects on traffic. We implemented a method to calculate the error between predicted and actual flight paths. Our concept is based on an air traffic scenario with a huge number of flights. Figure 1 summarizes the reporting process and describes the range of functions. The simulation generates a set of trajectories. The reference trajectory is simulated with the forecasted conditions and afterwards, the same flight is simulated again with varied conditions as actual trajectory. Both trajectory files include position, speed, heading, fuel, etc. information for each second. To speed up the calculation process, the error can be calculated at particular events (entry to terminal manoeuvring area (TMA), top of climb (TOC) or top of descent), in increments of $15 \mathrm{~min}$ between these events.

During upload to the database the required parameters are selected, transformed, and scaled to a common format. Based on the simulation data temporal and spatial deviation is calculated and values of key parameters are put into the statistics database. In the visualization the uncertainty area is displayed around the predicted path, see Figure 8.

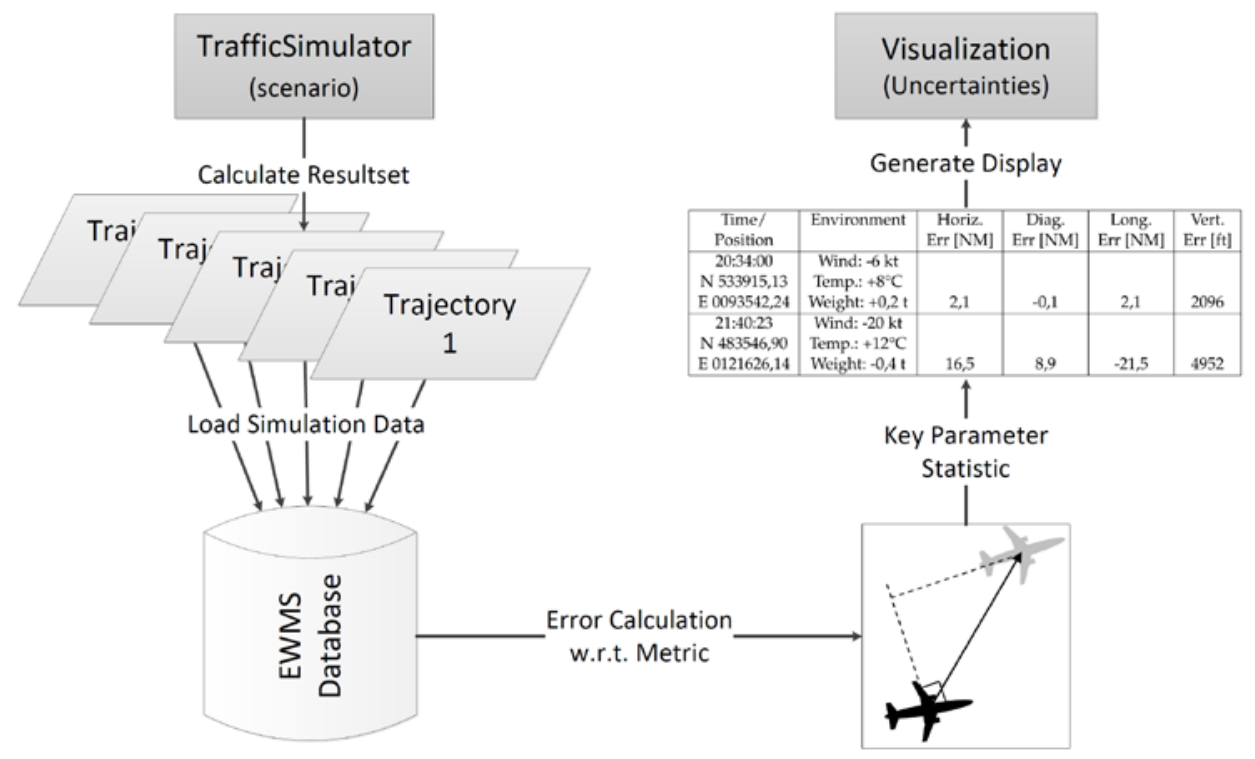

Figure 1. Reporting Process 
The position of a physical object can deviate from a predicted position in all four dimensions, specifically the three spatial dimensions and the temporal dimension. According to Knorr [4] figure 2 describes the spatial uncertainty at a specific timestamp as an ellipsoid. Vectors in the directions of the three axes (along-track, crosstrack, and vertical direction) define the size of the ellipsoid. The better the parameter forecast, the smaller the uncertainty area (blue line) which results in fewer conflicts.
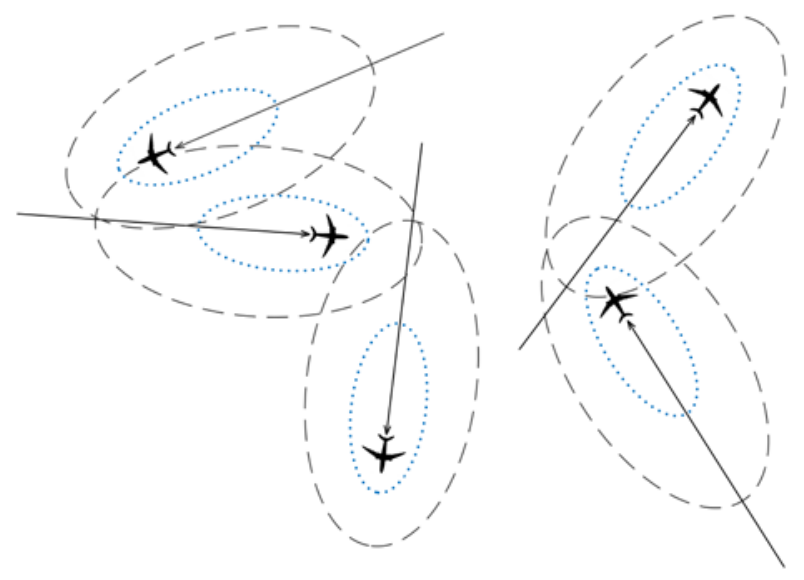

Figure 2. Uncertainty Visualization by ellipsoid

\section{Examined Parameters}

Deviations from a predicted flight trajectory can have several reasons. This section provides an overview of the most important parameters that are considered in this study. Firstly, aircraft performance has a significant influence on the flight profile. Especially the weight and the engine performance determine speed as well as takeoff and approach profiles. According to [9] a change of the aircraft weight has the most significant effect on trajectory generation. The actual aircraft weight is the amount of the operating empty weight, the weight of payload and the fuel weight. In the simulations of this study, the weight is changed by modifying the Base of Aircraft Data (BADA) [10] that is used by the simulator.

Furthermore, the temperature, wind direction and wind speed influence the ground speed and track of the aircraft [1]. According to the definition of ICAO [11], the standard temperature at mean sea level $T_{0}$ is $288.15 \mathrm{~K}$. The simulator used in this study generates the trajectory based on a particular meteorological grid file. The meteorological file defines a linear decrease of $0.65 \mathrm{~K}$ per $100 \mathrm{~m}$ increase altitude until reaching Tropopause at $11 \mathrm{~km}$. For higher altitudes the simulation assumes a constant temperature.

The last parameter considered in this study is navigation performance. Inaccurate measures in position can influence the quality of the actual flight route. ICAO developed the Required Navigation Performance Concept (RNP) to restrict the deviation caused by noisy navigation measuring [12]. The RNP specifies navigation categories for the required technical performance. For example RNP-0.3, RNP-4, RNP-10 and RNP-12.5 are currently used in practice. The value describes the maximum distance in NM of the measured position from the actual position. This deviation must not be exceeded for $95 \%$ of the whole flight.

The quality of the explained parameter forecast results in the quality of the certainty of the trajectory prediction. The next section describes which metrics are applied in the study to calculate the temporal and spatial uncertainty.

\section{Uncertainty Calculation}

The uncertainty in this work is distinguished in spatial and temporal errors. The spatial error is calculated with the Interval Based Sampling Technique [13]. This technique uses the temporal scanning method and has been developed by the FAA. Firstly, the position of two trajectories at the same time is selected. Secondly, the horizontal and the vertical distances between the positions are calculated. To determine the temporal uncertainty, the positions with the shortest distance are selected and the time difference is calculated.

\section{Spatial Error}

According to [14] Figure 3 illustrates the different vectors for error calculation. The horizontal error between actual flight position $\mathrm{F}$ and reference position $\mathrm{S}$ can be divided into the alongtrack error in flight direction and the cross track error that is the orthogonal distance between actual 
position and original intended direction of flight to the endpoint of the flight segment $\mathrm{E}$.

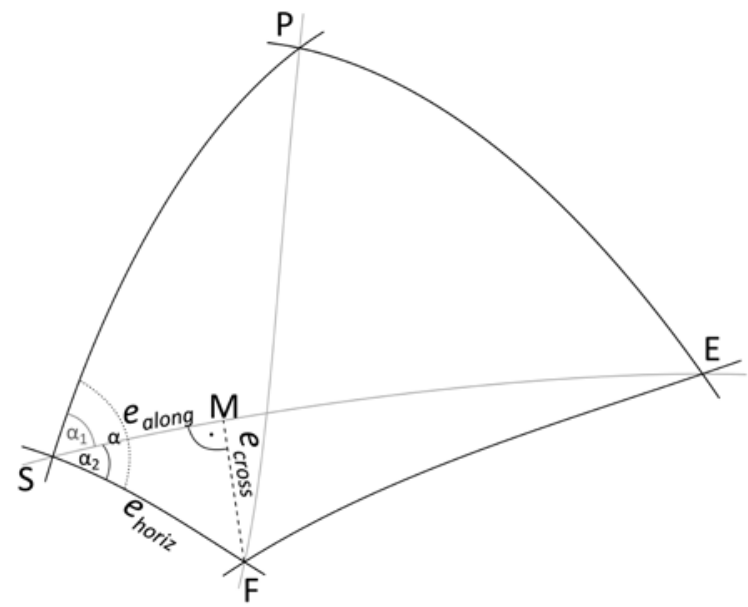

\section{Figure 3. Vector Metric for calculation}

The development of equations assumes that the earth is similar to a spherical object. Calculation of great-circle distance and angles between any two points on the surface of a sphere is based on spherical law of cosines. In the following, we develop the equations for the vector calculation. The latitude of a geographical position defines the location in relation to the North Pole. The angle between North Pole and Equator is $90^{\circ} \hat{=} \pi / 2$. Based on the Points $E\left(\varphi_{E}, \lambda_{E}\right)$ and $S\left(\varphi_{S}, \lambda_{S}\right)$ the edges $\overline{S P}$ and $\overline{E P}$ are represented by eq. 1 and 2 with $\varphi$ being the latitude and $\lambda$ is the longitude of the aircraft position.

$\overline{S P}=90^{\circ}-\varphi_{S}$

$\overline{E P}=90^{\circ}-\varphi_{E}$

\section{Horizontal Error ( $\mathbf{e}_{\text {horiz }}$ )}

The horizontal error is the distance $\overline{S F}$ between the predicted and actual position at time t. Eq. 3 is derived from the spherical law of cosines and the coordinates for point $S$ and $F$.

$$
\begin{aligned}
& \cos \overline{S F}=\cos \left(90^{\circ}-\varphi_{F}\right) \cdot \cos \left(90^{\circ}-\varphi_{S}\right) \\
& +\sin \left(90^{\circ}-\varphi_{F}\right) \cdot \sin \left(90-\varphi_{S}\right) \cdot \cos \left(\lambda_{F}-\lambda_{S}\right)
\end{aligned}
$$

Considering that $\cos \left(90^{\circ}-\varphi\right)=\sin \varphi$ and $\sin \left(90^{\circ}-\varphi\right)=\cos \varphi$ the equation specifies the edge $\overline{S F}$ in degree. To get the result in NM the distance is multiplied with the distance of $1^{\circ} \hat{=} 60 \mathrm{NM}$ :

$\rightarrow \mathrm{r}=60$

$$
\begin{aligned}
& |\overline{S F}|=r \cdot \cos ^{-1}\left(\sin \varphi_{F} \cdot \sin \varphi_{S}+\right. \\
& \left.\cos \varphi_{F} \cdot \cos \varphi_{S} \cdot \cos \left(\lambda_{F}-\lambda_{S}\right)\right) \\
& e_{\text {horiz }}=|\overline{S F}|
\end{aligned}
$$

\section{Along-Track Error ( $\left.\mathbf{e}_{\text {along}}\right)$}

The along-track error is the distance between planned position and intersection point of the orthogonal edge of the actual flight's position and the direction of flight. The distance of the segment $\overline{S E}$ can be calculated by eq. 6 and 7 .

$$
\begin{aligned}
& \cos \overline{S E}=\cos \left(90^{\circ}-\varphi_{E}\right) \cdot \cos \left(90^{\circ}-\varphi_{S}\right) \\
& +\sin \left(90^{\circ}-\varphi_{E}\right) \cdot \sin \left(90-\varphi_{S}\right) \cdot \cos \left(\lambda_{E}-\lambda_{S}\right) \\
& |\overline{S E}|=r \cdot \cos ^{-1}\left(\sin \varphi_{E} \cdot \sin \varphi_{S}+\right. \\
& \left.\cos \varphi_{E} \cdot \cos \varphi_{S} \cdot \cos \left(\lambda_{E}-\lambda_{S}\right)\right)
\end{aligned}
$$

For an entire derivation of metric values, the angles $\alpha, \alpha_{1}$ and $\alpha_{2}$ are calculated by eq. 8 to 11 . Equations 8 and 9 refers to triangles $\triangle S E P$ and $\triangle S F P$, in which all edges are known.

$$
\begin{aligned}
& \cos \alpha_{1}=\frac{\cos \overline{E P}-\cos \overline{S P} \cdot \cos \overline{S E}}{\sin \overline{S P} \cdot \sin \overline{S E}} \\
& \cos \alpha=\frac{\cos \overline{F P}-\cos \overline{S P} \cdot \cos \overline{S F}}{\sin \overline{S P} \cdot \sin \overline{S F}}
\end{aligned}
$$

For $\alpha_{2}$ in the triangle $\triangle S F M$ we have to make a 
case differentiation considering the longitudinal coordinates $\lambda_{F}, \lambda_{S}$, and $\lambda_{E}$.

- if $\lambda_{F}<\lambda_{S} \leq \lambda_{E}$ or $\lambda_{S} \leq \lambda_{E}<\lambda_{F}$ :

$$
\alpha_{2}=\alpha-\alpha_{1}
$$

- if $\lambda_{S}<\lambda_{F}<\lambda_{E}$ or $\lambda_{E}<\lambda_{F}<\lambda_{S}$ :

$$
\alpha_{2}=\alpha-\left(180-\alpha_{1}\right)
$$

Based on the angles it is possible to calculate the along track error in NM $(r=60)$ :

$$
\begin{aligned}
& \tan \overline{S M}=\tan \overline{S F} \cdot \cos \alpha_{2} \\
& e_{\text {along }}=|\overline{S M}|=r \cdot \tan ^{-1}\left(\tan \overline{S F} \cdot \cos \alpha_{2}\right)
\end{aligned}
$$

\section{Cross-Track Error}

The distance from the actual position to the segment line $\overline{S E}$ can be determined by eq. 14 and 15.

$$
\begin{aligned}
& \sin \overline{F M}=\sin \alpha_{2} \cdot \sin \overline{S F} \\
& e_{\text {cross }}=|\overline{F M}|=r \cdot \sin ^{-1}\left(\sin \alpha_{2} \cdot \sin \overline{S F}\right)
\end{aligned}
$$

Interpretation of the algebraic sign:

- $\alpha_{2}<0 \rightarrow$ The aircraft is north of planned trajectory

- $\alpha_{2}>0 \rightarrow$ The aircraft is south of planned trajectory

\section{Vertical Error}

The vertical deviation is the difference in altitude of the predicted trajectory and actual flight path. This error is described by a vector which is orthogonal to the xy-plane. If the vector is positive, the actual position is above the planned one. A negative vector means that the actual altitude is below the planned altitude.

\section{Temporal Error}

The temporal error describes the time difference between planned and actual flight position at a particular event. This metric can be applied to investigate the delay for arrivals or at TOC. The great circle distance is calculated with Vincenty's formulae [15] and starts with the time corresponding positions. The algorithm reads sequentially in the direction with the lower distance until the minimum is reached. For this error three different cases are possible:

- The time of the position with the minimum distance is before the reference time $\rightarrow$ the actual flight is premature.

- The time of the position with the minimum distance is after the reference time $\rightarrow$ the actual flight is delayed.

- The time of the position with the minimum distance is the same as the reference time $\rightarrow$ the actual flight is on time.

\section{Simulation}

For the measurement of the uncertainty investigation flight track data are needed. In this study the data are generated by fast time simulations. With the aid of fast time simulations complex traffic scenarios are analyzed. The simulation system models the aircraft performance, the environment, the pilot, as well as the controller. This fact makes an investigation of a huge scenario within a few minutes possible.

The traffic scenario is implemented and simulated with DLR's software TrafficSim [16] developed by the Institute of Flight Guidance. The simulation tool requires a flight plan, a constraint list including route, speed and other information for each flight, and the aircraft performance file (BADA 3.9) [10]. Beyond this, the mentioned meteorological grid file including barometric pressure, wind direction, wind speed, and temperature has to be defined in the settings (size of grid field is $1^{\circ}$ lat $\times 1^{\circ} \mathrm{lon}$ and $500 \mathrm{ft}$ ). The calculation of trajectories is based on the pointmass-model and logs the trajectory points in a file. The more sampling points, the more detailled is the uncertainty calculation. For our investigation we decided to log the pertinent information like time, coordinates, altitude, fuel flow, heading etc. every second. 
The best way to validate the deviations from a reference value is a lengthy trial. However, for the effect of vague environmental parameter forecasts, a simulated flight including all flight phases is sufficient. Thus, we implemented a simulation with a German domestic flight from Hamburg to Munich using an Airbus A320. The Aeronautical Information Publication published by Deutsche Flugsicherung (DFS) defines the standard routes in German airspace. According to the definition, we prepared the constraint list for the flight from Hamburg to Munich, see Figure 4. Eventually, the route distance of the mission is approximately 450 NM and includes not more than five routing points, which ensures flexibility for the trajectory generator. This implies that the waypoints can be overflown by mid of turn or start of turn with different turn radii (limited to 1-15 NM).

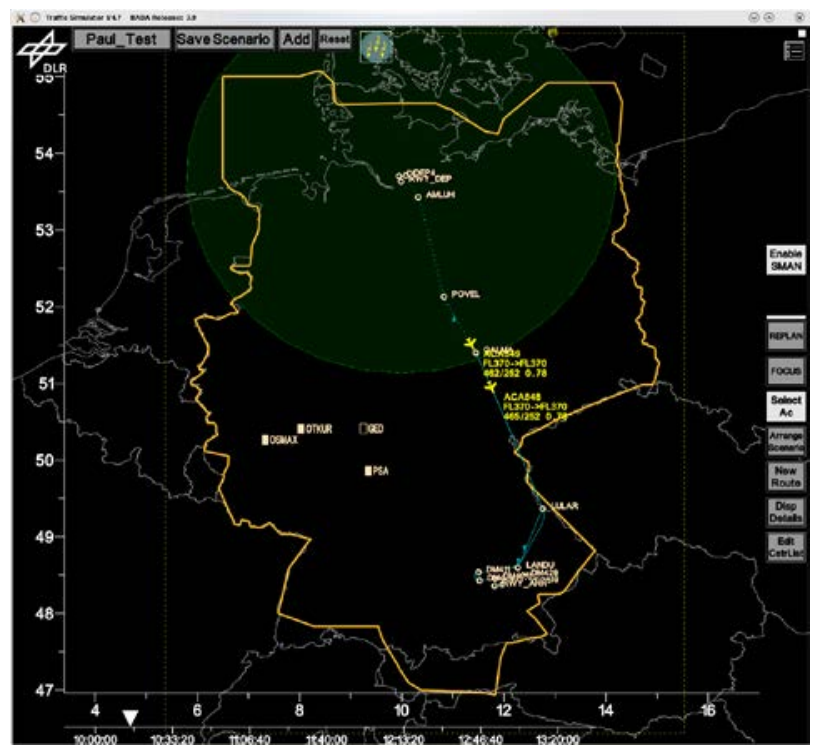

Figure 4. Arrival Route with Uncertainties

In order to simulate uncertainties in consequence of varied environmental parameters, we modified the input data and simulated the flight at a different day with the same take off time. Following list shows the crucial parameters and the range of modification:

- Wind direction: $0^{\circ}$ to $315^{\circ}$ (step range of $45^{\circ}$ )

- Wind speed at MSL: $0 \mathrm{kt}$ to $50 \mathrm{kt}$ (step range of $10 \mathrm{kt}$ )
- Temperature at MSL: $268 \mathrm{~K}$ to $308 \mathrm{~K}$ (step range of $10 \mathrm{~K}$ )

- Aircraft weight (in percent of MTOW): $50 \%$ to $100 \%$ (step range of $10 \%$ )

- True Airspeed: $\mathrm{Ma}=0.72, \mathrm{Ma}=0.78$ and $\mathrm{Ma}=0.82$

Applying the modifications, we get approximately 2,000 different trajectories for the flight with departure airport Hamburg and arrival airport Munich. In the context of the developed reporting process the set of trajectories is loaded to the database and the error based on the described metrics is calculated in the next steps. The following chapter illustrates the visualization and the acquired knowledge about the effect of an uncertain parameter forecast.

\section{Results}

\section{Visualization Framework}

During the preparation of the study we picked a set of frameworks, like Google Chart Tool ${ }^{1}$, JFreeChart $^{2}$, VisIt ${ }^{3}$, GeoTools ${ }^{4}$, NASA World Wind ${ }^{5}$ or $\operatorname{ArcGIS}^{6}$, that seemed to be convenient and investigated their features. As it turned out JFreeChart and NASA World Wind are the toolkits with the best documented java interfaces and opportunities for uncertainty imaging as well.

JFreeChart [2] is a free library for the Java platform and yields detailed value lists for quantitative analysis. Using the library it is possible to access data from any implementation of the defined interfaces and generate more than ten different chart types (pie charts, bar charts, scatter plots, etc.). Additional features include: export to PNG and JPEG image file formats, tool tips, interactive zooming or chart mouse events. To understand magnitude, dimensions, and relation, three-dimensional images in a geo spatial reference system are more convenient. We embedded a virtual globe in EWMS [6] to which we can easily

\footnotetext{
${ }^{1}$ https://developers.google.com/chart

${ }^{2}$ http://www.jfree.org/jfreechart

${ }^{3}$ https://wci.llnl.gov/codes/visit/about.html

${ }^{4}$ http://geotools.org

${ }^{5}$ http://worldwind.arc.nasa.gov/java

${ }^{6}$ http://www.arcgis.com/about
} 
add three-dimensional data objects. In NASA World Wind we can plot geometrical shapes like points, edges, polygons, cylinders, and ellipsoids. Furthermore, users can interact with the selected object by rotating it, tilting the view, and zooming in and out.

\section{Simulated Uncertainties}

All steps and features for the reporting process from figure 1 are implemented and usable. The following part describes knowledge gained by the simulation and reporting trials.

\section{Uncertainty caused by deviation of weight}

A variation of takeoff weight especially affects the vertical profile. As shown in Figure 5 the dark blue line is the reference flight $(75 \%$ of the MTOW). The light blue surface defines the vertical uncertainty. It can be easily seen that the error in weight forecasting predominantly influences the departure phase. The light red surface at the bottom is the projection of the profile to the reference zero level. When switching to the table view, the tool indicates numerical values and in this case maximum uncertainties of $8,000 \mathrm{ft}$ are calculated if the weight is $25 \%$ more or less than predicted with all other parameters remaining constant. After achieving the cruise level the vertical uncertainty decreases to zero.

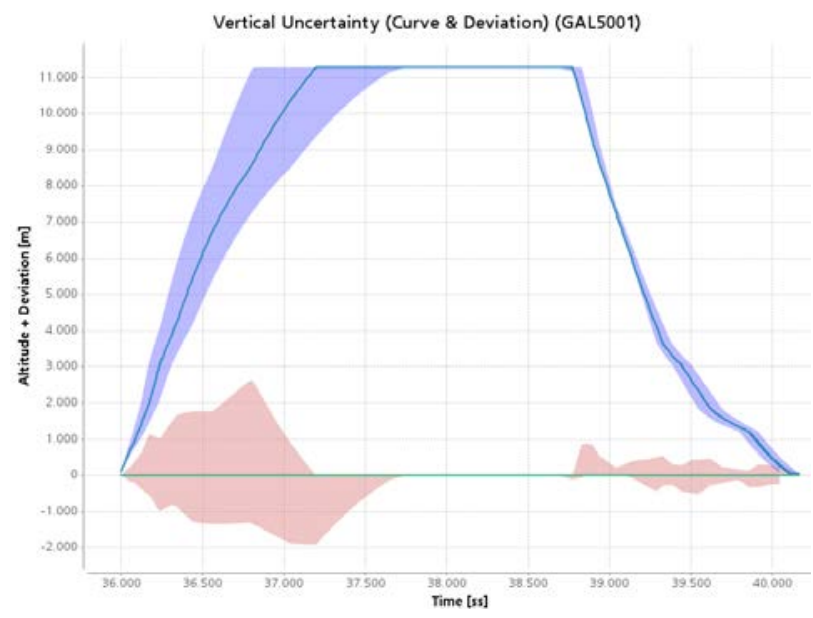

Figure 5. Vertical Error

The NASA display (Figure 6) shows the original track (green), the minimum altitude (blue), and the maximum altitude (red) with respect to the geographical location.

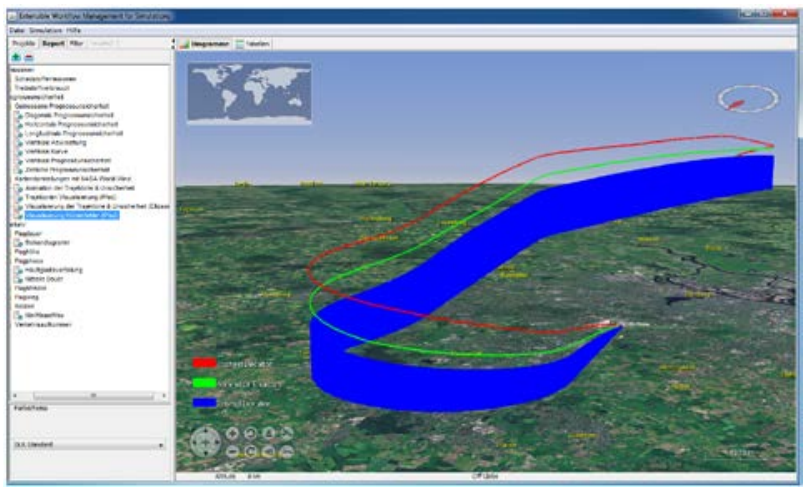

Figure 6. Vertical error in $N W W$-Display

\section{Uncertainty caused by deviation of speed}

A modified air speed affects hugely the duration of flight. Figure 7 displays the delay in positive y-direction and the earliness in negative $y$ direction. So, if the aircraft flies $\mathrm{Ma}=0.82$ it is $132 \mathrm{~s}$ earlier at the destination and $110 \mathrm{~s}$ delayed if it flies $\mathrm{Ma}=0.74$ instead of $\mathrm{Ma}=0.78$.

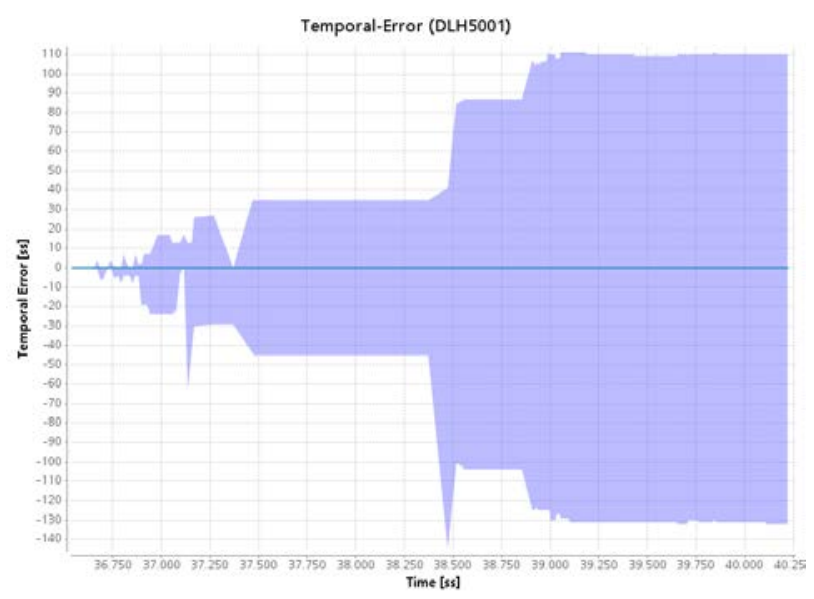

Figure 7. Temporal error

\section{Uncertainty caused by deviation of wind}

The example in Figure 7 represents the maximum along-track error if the wind direction is constant from $90^{\circ}$ but the true wind speed differs $\pm 10 \mathrm{kt}$ from the forecast. The Uncertainty increases continuously with flight duration at the beginning and remains constant from the middle of the flight. The conversion of the sign of value is because the position of the actual trajectory alters between west and east of the predicted position. The maximum deviation caused by wind speed is $3.5 \mathrm{NM}$. 


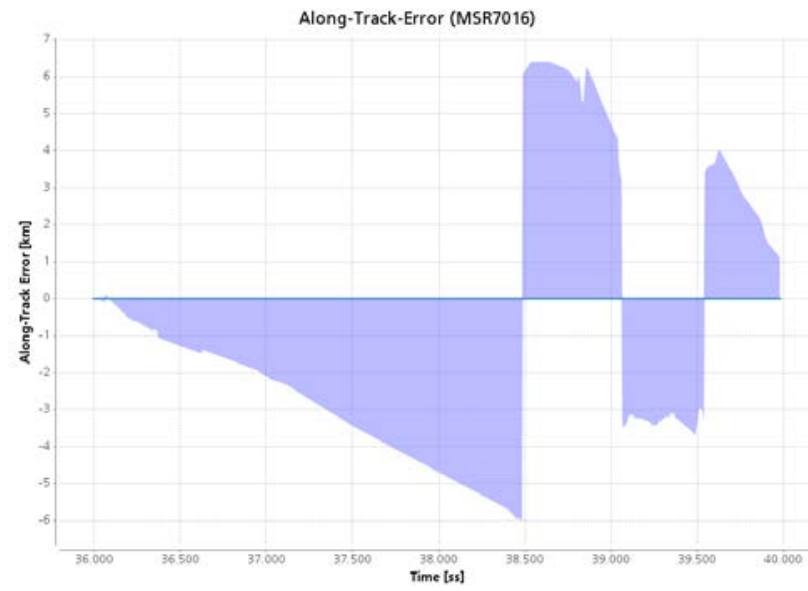

Figure 8. Along-track error

The effect of faulty wind direction forecast can be much greater than deviation in wind speed. In the selected example shown in Figure 8, the forecasted wind amounts to $30 \mathrm{kt}$ from east corresponding to $90^{\circ}$. The true wind arrives from $180^{\circ}$ (south direction). Since the flight from Hamburg to Munich is chiefly in southern direction, the wind changed from cross-wind to headwind. In case of headwind the ground speed reduces and the flight takes 271 sec longer than predicted. The headwind and longer duration has a direct implication to the along-track error. The spatial uncertainty increases to a maximum of approximately $25 \mathrm{NM}$.

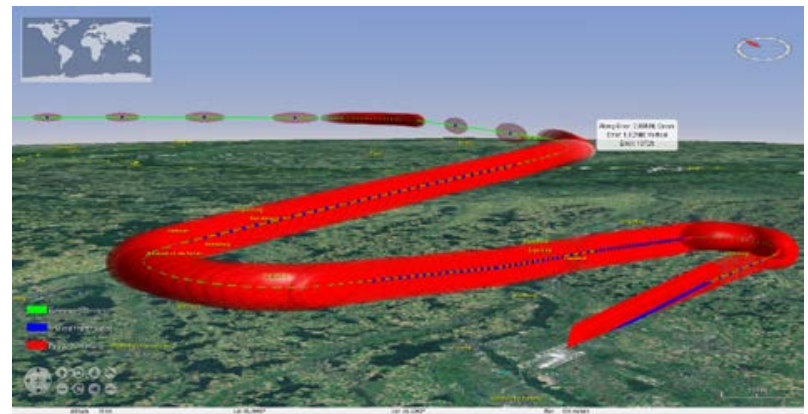

Figure 9. Arrival Route with Uncertainties

In Figure 9, the uncertainty is imaged in the ellipsoid shape as elucidated. In the Figure, the small blue dots are the logged positions of the predicted trajectory which are connected by a green line. Represented by the red ellipsoid the uncertain area can be seen. The sum of the sampling points and ellipsoids, at these points generate a kind of tube over the whole flight. This can be interpreted as the three dimensional space in which the aircraft will be.

\section{Uncertainty caused by navigation error}

In the scenario the error in determination of the position is simulated by a normally distributed shift of the trajectory as well as a variation of the turn radius. The developed display based on NASA World Wind in Figure 10 is characterized by the predicted green route and the distributed red deviations which are within a range of 4 NM (RNP4).

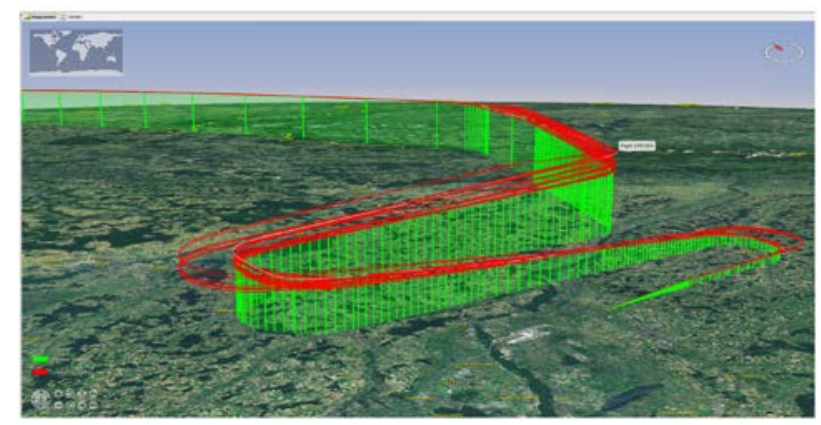

Figure 10. Navigation Uncertainties

\section{Conclusion}

The demand in civil aviation will increase in the future decades [17]. Infrastructure, runway systems and the airspace will reach its capacity limits. To be able to apply the improved trajectorybased air traffic management, investigations of the uncertainty of the predicted trajectory and decision support tools to visualize trajectories in combination with the deviation are necessary. The described concept is implemented in EWMS [6] and enables the user to load fast-time simulation results from the TrafficSim-tool [16], to calculate and compare trajectory uncertainties, and to visualize the results in JFreeChart diagrams as well as in a 3D NASA World Wind environment.

The developed calculation and visualization routines provide the ability to analyze trajectorybased air traffic management concepts, as well as support decision-making in air traffic control. It is conceivable to combine the visualization tool with models as developed by Wanke [8], to calculate the uncertainty in various traffic situations before takeoff. 
We analyzed the incluence of various environmental parameters on the precision of the trajectory prediction based on fast time simulations. The results show large vertical uncertainties with deviations of the weight, and horizontal uncertainties under different wind conditions. The true airspeed affects mostly the duration of flight. Deductive, we can resume that a more precise forecast of the influencing parameters aimed a smaller uncertainty and thus higher efficiency and safety.

In future, the tool will be used for other applications as e.g.

- mapping of sector complexity

- detection of conflicts in case of intersections of ellipsoids

\section{References}

[1] Kim, Jinwhan, K. Palaniappan, J. Thipphavong, 2004, Trajectory Uncertainty Modeling for Queueing Analysis of the National Airspace System, NASA Ames Research Center.

[2] Gilbert, David, 2009, The JFreeChart Class Library - Developer Guide, Object Refinery Limited, United Kingdom.

[3] Hogan, Patrick, 2009, NASA World Wind Java Software Development Kit, NASA Ames Research Center, http://worldwind.arc.nasa.gov.

[4] Knorr, David, L. Walter, 2011, Trajectory Uncertainty and the Impact on Sector Complexity and Workload, Federal Aviation Administration (FAA), Deutsche Flugsicherung GmbH.

[5] Bronsvoort, Jesper, G. McDonald, R. Porteous, 2009, Study of Aircraft Derived Temporal Prediction Accuracy using FANS, Airservices Australia.

[6] Scharnweber, Alexander, S. Schier, 2011, Automated Simulation Data and Process Management for Heterogeneous Air Traffic Management Simulations, German Aerospace Center (DLR), Germany.

[7] Mueller, Tysen, J. Sorensen, G. Couluris, 2002, Strategic Aircraft Trajectory Prediction Uncertainty and Statistical Sector Traffic Load Modelling, Seagull Technology, Inc., Campbell.
[8] Wanke, Craig, M. Callaham, D. Greenbaum, 2003, Measuring Uncertainty in Airspace Demand Predictions for Traffic Flow Management Applications, The MITRE Corporation Center for Advanced Aviation System Development, McLean.

[9] Temme, Marco-Michael, et al., 2007, ATMTechnik in Gegenwart und Zukunft, German Aerospace Center (DLR), Germany (in German).

[10] Poles, D., 2009, Base of Aircraft Data (BADA) Aircraft Performance Modelling Report, Eurocontrol Experimental Center.

[11] ICAO, 2007, Meteorological Service for International Air Navigation, Annex 3, International Civil Aviation Organization.

[12] FAA, 2007, Instrument Procedures Handbook, Federal Aviation Administraion.

[13] Paglione, Mike, Robert Oaks, 2007, Implementation and Metrics for a Trajectory Prediction Validation Methodology, William J. Hughes Technical Center, Atlantic City.

[14] Mondoloni, Stephane, Sipke Swierstra, Mike Paglione, 2005, Assessing trajectory prediction performance - metrics definition, CSSI Inc., Washington.

[15] Vincenty, Thaddeus, 1975, Direct and Inverse Solutions of Geodesics on the Ellipsoid with application of nested equations, Ministry of Overseas Development.

[16] Edinger, Christiane, Angela Schmitt, 2012, Rapid Prototyping for ATM operational concepts development, German Aerospace Center (DLR), Germany.

[17] Eurocontrol, 2010, Long-Term Forecast: IFR Flight Movements 2010-2030, EUROCONTROL.

\section{Email Address}

Paul.Weitz@dlr.de

\section{Integrated Communications Navigation and Surveillance (ICNS) Conference}

April 23-25, 2013 